\title{
Usability Study of a comprehensive table tennis AR-based training system with the focus on players' strokes
}

\author{
Ayman Nabil' ${ }^{1}$, Habiba Hegazy ${ }^{1,}$ *, Mohamed Abdelsalam¹, Moustafa Hussien ${ }^{1}$, Seif \\ Elmosalamy $^{1}$, Yomna M.I. Hassan ${ }^{1}$, Ayman Atia ${ }^{2,3}$ \\ ${ }^{I}$ Faculty of Computer Science, Misr International University, Egypt \\ ${ }^{2}$ HCI-LAB, Faculty of Computers and Artificial Intelligence, Helwan University, Egypt \\ ${ }^{3}$ October University for Modern Sciences and Arts (MSA), Egypt
}

\begin{abstract}
Table tennis game is based on the speed of the player's response to different attacks and defense strokes. A way to enhance the player's performance and technique while training is to update the player with the mistakes in real-time. This paper presents a system that focuses on detecting the correct and wrong strokes within the following stroke types: Forehand drive, backhand drive, and forehand topspin. By the usage of Augmented Reality, the system helps the players to get their results and direction easily using AR-based mobile application when practicing real-time. A usability study has been made to measure the learning style of the players by letting the players train on different strokes with the system. Moreover, an experiment has been done to measure the efficiency of the application and compare different algorithms to overview their performance in identifying the strokes based on accuracy and time taken.
\end{abstract}

\section{Keywords: Stroke Classification, Stroke Identification, Table Tennis, IR Depth Camera, Hand Gestures, Augmented Reality}

\section{Introduction}

With the presence of modern technologies like smart bands, mobiles and, advanced glasses, technology become an incredible aspect of our everyday. In sports particularly, technology is utilized to help increase the player's performance and playing technique through the training sessions. Sensors were used to help in detecting the player's motion and strokes. Nowadays, virtualization technology such as Virtual Reality, and Augmented Reality headsets are integrated into the sports field to notify players and improving their training environment. In table tennis, technology was investigated in the creation of the electronic scoreboard, automated scoring system, interactive ping pong table, and high-speed camera's and recently, technology was shown to create robots to compete with. On the other hand, any technology that can be created to points on the player's correct and wrong movements to predict or measure the player's performance will be very useful [1]. This makes the human-computer interaction in table tennis an important research field and important to increase the player performance and speed. According to statistics made in the United States in 2017, there are 16.04 million players around the world play table tennis [2]. This paper focuses on three different types of strokes such as forehand topspin, forehand drive and, backhand drive movements.
One of the basic table tennis strokes is a forehand drive. It is a stroke of an attack with a little percentage from the topspin stroke. It is performed as a reply for medium or long-range topspin or ball drifts. According to Ben Larcombe [3] a forehand drive should be done correctly on six steps: The racket should be ranging between the waist height, close the racket angle a little bit, lean backward from the waist by a small amount, when the ball arrives, turn your racket and move it forward towards the table and up, contact the ball instantly when it comes next to your body, the touch would have been very smooth, just in the center of the racket. These steps are the same for either a forehand drive or backhand drive. On the other hand, a common mistake is putting your arm across your body on the follow-through this is going to slow your recovery for the upcoming shot as shown in fig. 1 (a).

The backhand drive stroke is one of the most important strokes in table tennis sport, according to Killertips Network, L. Sharon, and W. Suwito [4] there are four sections for the backhand drive; the backswing, the stance, the finish, and the strike. Mainly the stance consists of positioning your feet, knees, body, and arms to get started to the shot. The backswing is about keeping your hand in the right place and placing the racket in front of your belly. The strike is the part where the player starts hitting the ball as if it's the topspin to create some

${ }^{*}$ Corresponding author. Tel.: +201094586906

E-mail: habiba1611146@miuegypt.edu.eg

(C) 2020 International Association for Sharing Knowledge and Sustainability.

DOI: 10.5383/JUSPN.13.01.001 
momentum. Lastly, the finish where the bat should be in front of the player's chin. Players usually make some common mistakes while playing a backhand drive such as playing the stroke as a short shot and standing directly behind the ball while it's coming as shown in fig. 1 (b).

The forehand topspin stroke is a complete attacking stroke where it takes all the power from the player to get the ball to the maximum speed possible to make one of two things, to make it harder on the opposite player to block or to use it to block an opponent spin. The technique used to make a correct forehand topspin $[3,4]$ : Begin with your racket under waist height, close your racket slightly, twist your knees, and lean backward from your lower body. As the ball gets to you, push your legs up, twist forwards, and accelerate your racket upwards. One of the common mistakes for doing a topspin is either close to the racket angle or open it just before ball contact, which means that the racket angle is not correct, each stroke is different. This leads to inefficient play with very bad stability in the player's hands as shown in fig. 1 (c).

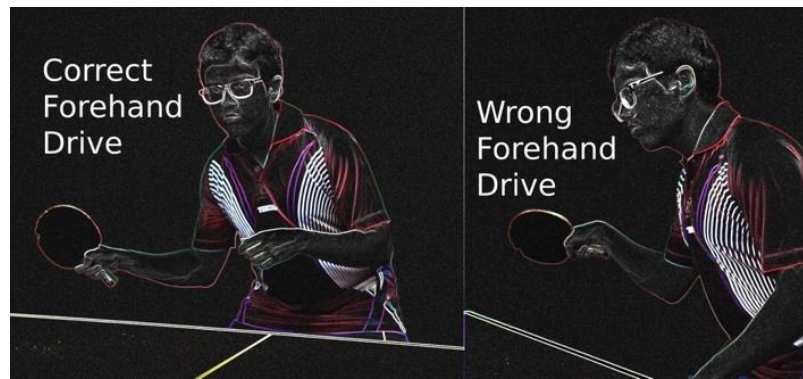

(a) The correct and wrong techniques while the player was performing forehand drive stroke.

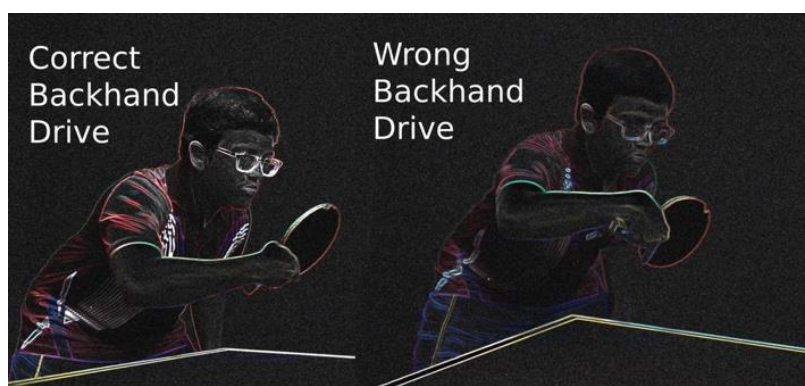

(b) The correct and wrong techniques while the player was performing backhand drive stroke.

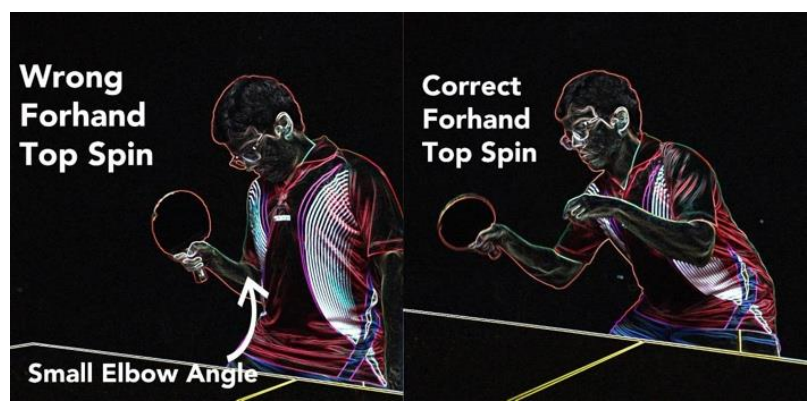

(c) The correct and wrong techniques while the player was performing forehand topspin stroke.

Fig. 1. Clarification of table tennis strokes techniques during the game.
This paper is an extension work for our previous papers [5, 6], the main contribution of this paper relies mainly on classifying the different stroke types (correct/wrong) through various algorithms. Also, to support the system with an AR notification system to increase the table tennis player's skills to the next level by giving the users real-time performance and results. Also, we aim to measure the learning style of the players by undertaking a usability study.

The paper is partitioned into four sections. Firstly, the related work where we present other researches in different domains related to the techniques used in the proposed system. Secondly, the methodology where we mainly explain the proposed approach and the techniques used. Then we go to the experiment where we show the proposed system contribution and results. Fourthly, a discussion where we investigate the basis of the introduced platform. Finally, summary and future studies where the results and outcomes of the research are presented.

\section{Related Work}

This section is split up into various parts. Each part is required to indicate how it is used in our framework.

\subsection{Systems of table tennis}

In this sport, movements are categorized into either primary and advanced stages. Drive, topspin, and push are crucial basic strokes. It can determine and identify tennis strokes by capturing the motion of the hand. The research [7] was used to assign a hardware component to the racket to track player movements. Some studies achieved $95.7 \%$ accuracy [8] by the use of this methodology. By thus the existence of wearable devices such as wearable IMU systems and utilizing basic equations [9] reached a significant classification result for table tennis serves, forehand, and backhand strokes.

The classification of ball spins was mentioned in another research. Many kinds of strokes lead to many spins and speeds of the ball. [10] offered a ball speed and spin system, inserted within the table racket, with a single IMU, researchers reached a precise of $79.4 \%$. In a different study, a robot has been made by the researchers that can compete with a human player [11]. Therefore, detection and classification of the strokes in table tennis are known to be a hand motions problem based on hand movements as table tennis sport.

\subsection{Hand gestures}

In human-computer interaction, the recognition of hand motions is considered to be a genuine domain. It provides multiple techniques that are considered close to the behavior of humans [12]. Gestures act as a very significant and efficient technique, especially through sport training applications, Augmented Reality, and online environments. Using Kinect and SVM algorithm, [13] offers a suitable solution to weighty HCI interfaces in real-time which results in $95.42 \%$ accuracy. Authors in the [14] developed a multi-core DTW solution, 0.28 -second time-consuming, multi-core algorithm. Hand movements to be observed will be recorded. In detecting movements and actions of the body the sensors play a significant function $[15,16]$. IR Depth camera is one of these sensors. 


\subsection{Background of the IR depth camera sensor}

The idea of smart technology involves the use of knowledge in various technologies and areas. Sports are one of these areas. Consequently, people seek to efficiently utilize technologies to increase sport competitive environment. The identification and recognition of the player's movements are the primary goals of the development aspect of the sport. Detection of movements achieved generally using a camera or wearable sensor movement devices in a specific IR depth camera. The use of IR depth cameras like Kinect which will be a vital role in the sports field in the future such as folk dance [17]. In [18] authors used the SVM algorithm and IR depth camera to identify the human posture to reach optimal accuracy for various positions. The results indicate that Kinect can identify various positions in high accuracy with a low-cost device. In addition, [19] research has pointed out how Kinect SDK can identify joints correctly and it has proposed an AR judo training program. The use of IR depth cameras including Kinect also plays a leading part in the development of the sports sector.

Neither of the previous studies stated that an IR depth camera had been used in table tennis, relying on player motions for the optimal performance. The paper has a maximum exactness of $96.29 \%$ [20] instead of an IR-depth camera used for the performance of the player when balls are received. A large amount of noise is often produced by the usage of the IR depth camera which affects data collection and consequently the system classification [21]. Therefore, the filtering phase with sensors is an essential prerequisite.

\subsection{Pre-processing (Filters)}

Tools like IR-depth cameras and other sensors identify data with a huge background noise [21]. Although there's a real need to remove noise, due to the light systems, and the data is encircled by noise. The bilateral filter for the joint was used for the identification of captured images in order to improve image quality [22]. [23] used the Gabor Filter with the SVM algorithm to resolve a hand motion detection procedure which removed lighting limits. In addition, the Gabor filter improved the accuracy of the recognition. Moreover, Nuttaitanakul and Leauhatong [24] utilized the channel length for human action characterization filtration. On the other hand, the Kalman filter has been used to monitor the body joints to remove the noise of the unwanted vibrations and thus decreases the variation in the center of joint position [25]. Furthermore, the Kalman filter contributed by dismissing signals to create a moving object position and orientation in a real-time application using sensor motion devices which affect signal reliability [26]. The main aspect left is to identify the movements after recording the gestures of table tennis utilizing the IR-depth camera. One of the most significant classifiers is the dynamic time warping algorithm for real-time systems.

\subsection{Hand Gesture Algorithms}

By the usage of the signal taken from the IR depth camera, we need to classify the movements and strokes made by the player. Numerous and various algorithms were utilized in various hand signals application. The study [27] presented an android application online for evaluating the strokes performed mounting the smartphone to the player's wrist using the decision tree algorithm, reaching to $77.21 \%$ and $69.63 \%$ on average accuracy. According to Chao Xu et al., [28] they tested the different algorithm on Finger-writing with Smartwatch system, reaching a result that out of naïve bayes, logistic regression, and decision trees, logistic regression was the best classifier with $99.20 \%$ and $97.10 \%$ for detecting finger and hand movements sequentially. Also, the use of the Random Forest algorithm was used for automatic hand motion recognition where authors reached an accuracy of $98 \%$ [29]. The usage of a convolutional neural network (CNN) algorithm was mainly used in the sport in the field of image processing. [30] represented a real-time table tennis forecasting system, the usage of $\mathrm{CNN}$ was to do the $3 \mathrm{D}$ pose estimation with the usage of an RGB camera. Moreover, the study [31] suggests a quite effective online classification and recognition method of null or negative lateness in hand movements by the usage of CNN.

In addition, Dynamic Time Warping (DTW) examines the appropriate resemblance among two-time series with particular limitations. Any information can be analyzed using DTW, which can be converted into a linear sequence. They utilized dynamic time warping and IR-depth camera in activity recognition for human development because of their solidity of pace or design while executing the tasks $[32,33]$. In another instance, the usage of a smart band that consists of an accelerometer and gyroscope with the DTW algorithm [34]. The authors of [35] presented a system to train tennis shooting data on two levels of hierarchical classification based on the principle of using the DTW and QDTW. [36] Authors used Microsoft Kinect to develop the recognition of hand movements using DTW and HMM algorithms. Researchers found that at the moment of the consideration of a requirement, DTW was a better option than HMM.

Due to the FastDTW appearance, the speed and accuracy of systems had been different [37]. This has assisted other applications to reach 98\% accuracy in real-time. Authors in [38] introduce a methodology that optimizes the concept of athlete coaching through the use of an IR camera to recognize the athlete's wrong joints and notifies him/her prior to the occurrence of the injury. Their outcomes indicate that the approach of FastDTW has surpassed different methods and may achieve a $98 \%$ accurate recognition of dependent user gestures. The classified data would be used to get information to the user through an interface, one of those interfaces is augmented reality.

\subsection{The use of Augmented Reality in hand gestures}

Augmented Reality can turn into the irreplaceable apparatus which will be utilized by all players to get constant information about each activity or movement they make. The usage of Augmented Reality with self-directed sessions, sustained guidance, and feedback in training or learning sessions can improve the playing style and performance [39]. Authors in [16] suggested an AR framework, primarily in sports, that provides guidance and feedback. The major difficulties in the area of hand gestures would be to have the best classification accuracy, as the variety of other ways people perform gestures with different speed levels.

Moreover, authors of [40] proposed an improved 3D ping pong platform for two players through Wi-Fi access called ARPP. Where the players can control the rackets by moving their smartphones. In the volleyball sports field, the study was made to evaluate and analyze the load of the knee for the players in volleyball by jumping into Augmented Reality (AR) during preserving the perceptive associations by repeating the 
visual characteristics of the court of volleyball. The study shows that $\mathrm{AR}$ can be used to better replicate competitive information from a clinical assessment [41]. Besides, a research study made to examine the boundaries and plan prerequisites for creating a headset for downhill winter athletes for Augmented Reality that can enhance spatial-awareness, visual perception, and lessen injury [42]. However, AR was also used for navigation in lots of systems. Where authors [43] proposed a system based on a usability study for Indoor Library Navigation, they used AR technology to quickly enhance user functionality and knowledge inside the library in the right direction.

\section{Methodology}

The system consists of several sections. Firstly, the collection of data using an IR depth camera, in which data is collected for different joints. Secondly, a pre-processing section consisting of the segmentation of strokes and the filtration of data. Thirdly, the feature extraction section for the identification of critical data features. Fourthly, the processing section where the strokes are classified. Finally, the interfaces showing how data is displayed on both mobile devices and augmented reality as shown in fig. 2 . point and the following points to which the player is allocated The Euclidean distance is utilized in identifying and detecting any stroke from the sensor sequence. The length of the section which linked the two points is tested by the Euclidean distance.

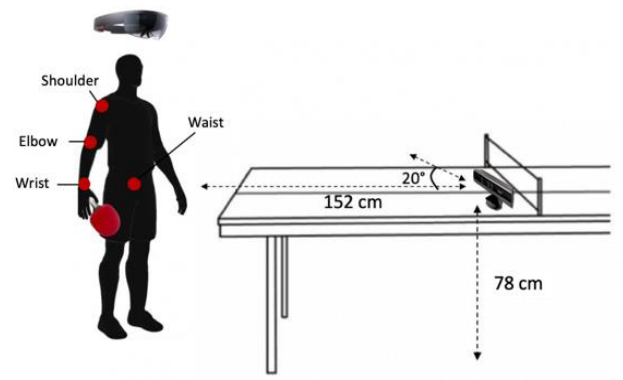

Fig. 3. The framework tracks and records the player's joints and its developments identified with table tennis strokes

\subsubsection{Data Filtering}

The Kalman filter is used for removing noise from the IR depth camera. For noise reduction and movement tracking Kalman filter preferred. It is used to improve the precision of the IR depth measurement and shows an appropriate pre-

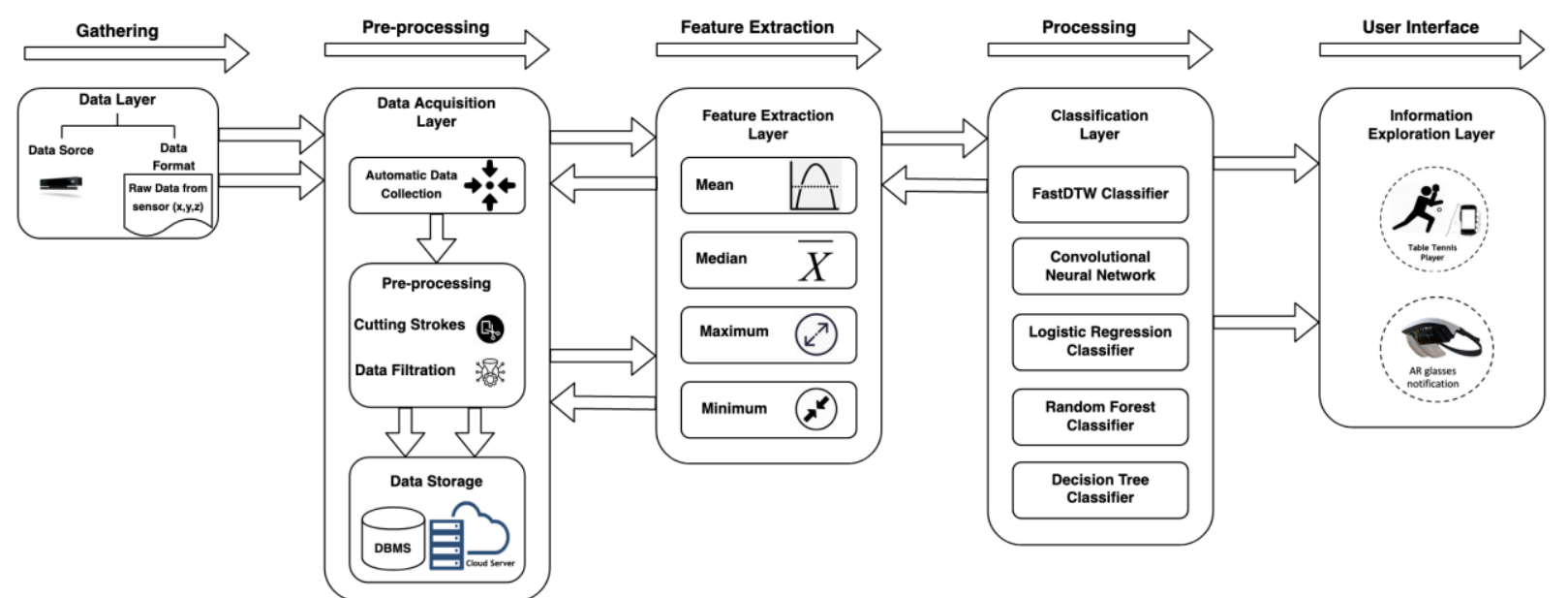

Fig. 2. Block technical diagram and system architecture.

\subsection{Data Acquisition}

The acquisition of the data is performed by utilizing the IR depth camera SDK, which differentiates different joints (elbow, shoulder, wrist, and waist) as shown in fig. 3 from the player's body. This approach of data gathering wasn't affected by environmental factors. The IR depth camera receives threedimensional points $(\mathrm{X}, \mathrm{Y}, \mathrm{Z})$, for each joint. The obtained data is transferred to the inner room server linked to the IR depth camera.

\subsection{Pre-processing}

This phase involves filtering, segmenting, and modifying data collected by the sensor just before the phase of processing.

\subsubsection{Stroke Segmentation}

After data acquisition, the stroke should be segmented from the collected source of data. In table tennis strokes, the system measures the Euclidean distance between the main starting processing and precise change, as described in $[25,26]$. Due to the high-frequency variations in the joint location and skeleton extraction, two forms of noise impact the IR camera depth time series results. Our obtained data is passed through the Kalman filter to enhance the IR-depth camera position estimation and to remove the noise affecting data such as temperature change, gravity, vibration, etc.

\subsubsection{Feature Extraction}

Extraction of the features is an important phase before the classification of the data and provides more information about the data and the mechanism. It gives us more understanding of the data and increases the performance and accuracy of learning algorithms [44]. Through this stage, the perception of the gathered data and the initial raw data set was decreased for the processing phase to more manageable and related categories. Multiple feature extraction techniques are used to decrease the number of resources available for evaluation without lacking significant or specific information and classify essential data characteristics. Multiple feature extraction techniques were used such as mean, median, minimum, and maximum. 


\subsection{Processing}

After the pre-processing phase, the collected data is being labeled using FastDTW. It is an analysis of time series and algorithms used to calculate the connection between two separate time and speed sequences. FastDTW provides nearoptimum alignments to DTW and $\mathrm{O}(\mathrm{N})$ complexity of time and memory that requires $\mathrm{O}\left(\mathrm{N}^{2}\right)$.

FastDTW was developed on the basis of Chan and Salvador [37], utilizing a multi-level approach with three main operations. FastDTW is really useful. First, the coarsening of the data points to the time series. Second, a prediction is primarily found on a minimum distance warp trajectory in the lowest part of the stroke flow. Finally, optimizing the disruption path generated by local warp trajectory alterations from the lower part of the stroke current. FastDTW decreases the time series in DTW by this approach.

First, a cost matrix begins to be established between the player's stroke and every stroke in the dataset. FastDTW begins to calculate the initial value on a matrix between the stroke points and each stroke point of a dataset with the two points and the neighbor.

Second, The FastDTW uses backtracking of the greedy search and the cost matrix to only reach the gap through the two strokes. In addition, FastDTW typically begins to distinguish among these two strokes by adding the element to a cost matrix at the top left. The algorithm ensures that each stroke in the data set reaches a balance of the distance from the test stroke. Eventually, FastDTW starts to search for the optimal distance of equilibrium. Thus, the label of the stroke may be identified from the dataset.

Although, in order to test the efficiency of FastDTW algorithm, we compared this algorithm will logistic regression, random forest and decision tree which were implemented in their default structure. Moreover, we compared the FastDTW algorithm with the Convolutional neural network algorithm which was implemented by the model presented in the research presented by Devineau et al., [45]. The model is based on using CNN classifying dimensional data and not depending on image processing.

\subsection{Augmented Reality Interface}

Augmented Reality (AR) is a technique that deals primarily with the graphics, sound, video, and many other sensor-based data on physical objects, utilizing the camera interface and using the computer-based vision recognition algorithms. By the usage of AR, the system can give real-time feedback to the player instead of the old technique used of recording the training session and then entering the video recorded into software that analyzes their sessions and get them a report of how many wrong and correct strokes they have done.

The Augmented Reality was built to give certain notifications for the player such as: if the stroke is wrong or correct, and if the stroke was wrong the system notify the joint where the mistake took place as shown in fig. 4. In the experiment section, we will test the usage of Augmented Reality and make a usability study on learning style with the players which tried the system.

\section{Experiments}

In this part, we set out the environment needed, and we collected the dataset. We present the algorithm comparison for the stroke classification in our system. Also, we present an experiment on usability study divided into two sections the usage of AR and the learning style of the player.

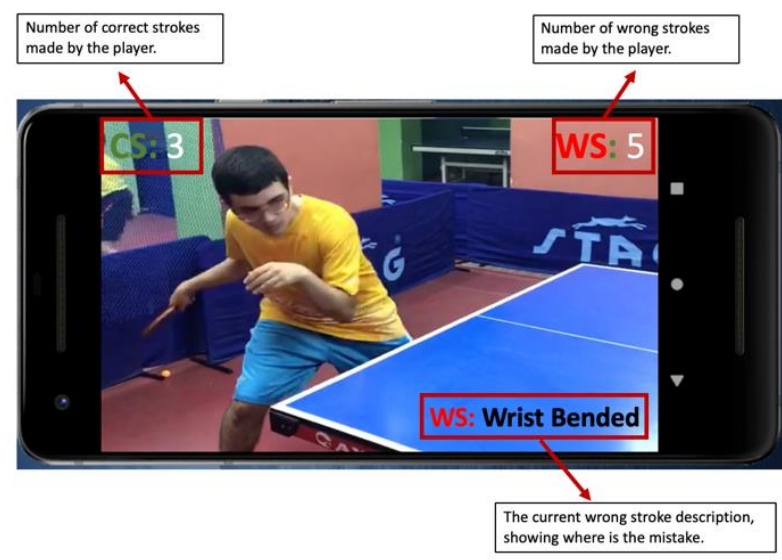

Fig. 4. AR display screen to notify players while training

\subsection{Equipment Setup}

With the original IR depth camera in the proposed platform, gesture recording was accomplished. The IR depth camera was utilized to monitor $\mathrm{X}, \mathrm{Y}$, and $\mathrm{Z}$ directions and its timestamps of four joints of the skeleton. This camera is placed on the table, $78 \mathrm{~cm}$ from the ground while the player is set to be $152 \mathrm{~cm}$ away as shown in fig. 3 .

\subsection{Data Collection}

Overall, around 1200 trials were collected on three main strokes: forehand drive, backhand drive, and forehand topspin, from five professional players (three males and two females). Every player had to repeat 80 times every stroke with a total of 240 trails per player. Testing is equally divided into correct and incorrect strokes.

\subsection{Experiment (1) - Stroke Classification Accuracy}

In this experiment, our system evaluated FastDTW, CNN, Logistic Regression, Random Forest, and Decision Tree algorithms for detecting and classifying the three main strokes of table tennis performed correctly and incorrectly. We used 150 samples for training and 50 samples for testing for each correct/wrong stroke with a total of 900 samples for training and 300 for testing. We also calculated the time that it is taken by each algorithm for classifying a single played stroke. The results of the algorithms are shown in table 1.

\subsection{Experiment (2) - Usability}

The usability experiment was done with the help of 50 different participants in the age group of $(13-28)$. The percentage of females where $45 \%$ and males where $55 \%$. Moreover, $60 \%$ of the players where international table tennis 
Author et. al. / Journal of Ubiquitous Systems \& Pervasive Networks, 1 (2020) 01-09

Table 1. Different classification algorithm comparison

\begin{tabular}{|c|c|c|c|c|c|}
\hline & FastDTW & CNN & $\begin{array}{c}\text { Logistic } \\
\text { Regression }\end{array}$ & Random forest & Decision Tree \\
\hline Correct backhand drive & $94 \%$ & $96 \%$ & $86 \%$ & $78 \%$ & $80 \%$ \\
\hline Correct forehand drive & $98 \%$ & $98 \%$ & $86 \%$ & $82 \%$ & $78 \%$ \\
\hline Correct forehand topspin & $94 \%$ & $92 \%$ & $84 \%$ & $78 \%$ & $76 \%$ \\
\hline Wrong backhand drive & $90 \%$ & $92 \%$ & $80 \%$ & $80 \%$ & $72 \%$ \\
\hline Wrong forehand drive & $96 \%$ & $96 \%$ & $86 \%$ & $86 \%$ & $78 \%$ \\
\hline Wrong forehand topspin & $92 \%$ & $92 \%$ & $82 \%$ & $74 \%$ & $70 \%$ \\
\hline Average Accuracy & $94 \%$ & $94.33 \%$ & $84 \%$ & $77.33 \%$ & $75.67 \%$ \\
\hline Average time (single stroke) & $0.98 \mathrm{sec}$ & $2.15 \mathrm{sec}$ & $1.73 \mathrm{sec}$ & $2.08 \mathrm{sec}$ & $0.99 \mathrm{sec}$ \\
\hline
\end{tabular}

players and $40 \%$ where national table tennis players.

Participant's preferences were indicated as to the number of questions they were asked. Firstly, we asked them to give us a brief about them and their expertise in table tennis. Secondly, they started rating the system from the weakest points to the strongest, covering all the critical and major usages of the system. Such as performance, complexity, setup, destructibility, comfortably, and the whole experience with IR depth camera and AR glasses.

\subsubsection{Usage of Augmented Reality}

Overall, users' perceptions of the performance usability of the system were very positive. The usage of AR was reported as highly comfortable 40 of 50 of the players were very satisfied as shown in fig. 5 (b). Moreover, as AR is a head-set wearable device. we focused also on is the AR screen display is distributive or not. We came out with a result that 36 out of 50 players agreed that it was not distributive as shown in fig. 5 (a). Participants also indicated that they would not hesitate to wear the AR head-set and that they did not experience any negative reactions while wearing it. Moreover, their feedback on the navigation on the AR that was very reasonable and satisfying to them.
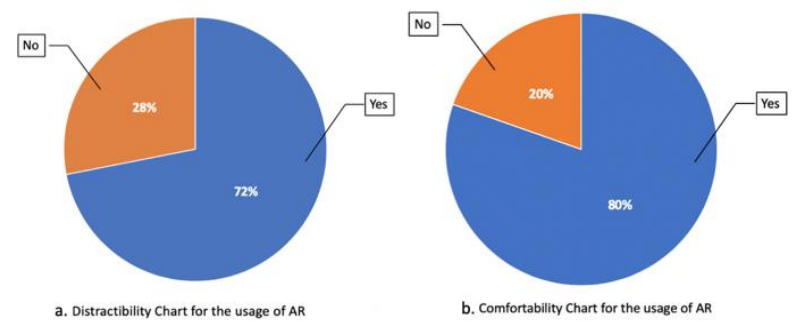

Fig. 5. Players' opinions for the usage of Augmented Reality in the sports field

\subsubsection{Learning Style}

We constructed an experiment that shows how well the system improves the playing style of the players. In this experiment, we asked 20 beginners' players to perform the three different strokes of the table tennis (forehand topspin, forehand drive, and backhand drive) in all of the three sessions. Each session contains the average percentage of mistakes made by players on all three strokes. Each player played the three strokes around 25 times by focusing on improving the style according to the system instructions. The average number of mistakes is not the direct number of mistakes. The aim was to measure the enhancement of the player at each stroke.

As shown in graph fig. 6 the number of mistakes produced by the players decreased by each session. Moreover, we asked the users to answer a survey question whether their performance enhances and $90 \%$ concluded that the system did enhance their learning curve.

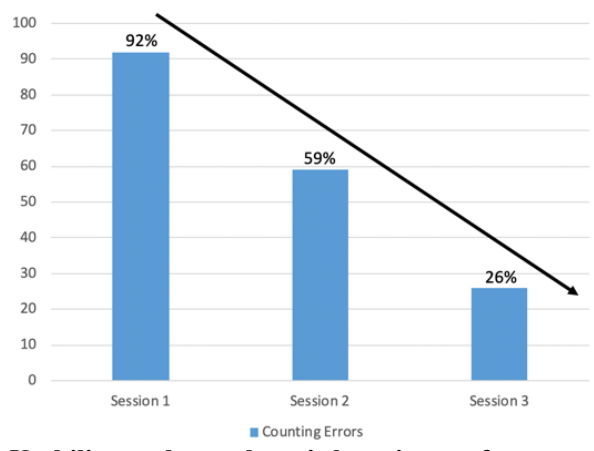

Fig. 6. Usability study on player's learning performance

\section{Discussion}

According to the results shown in table 1 illustrates how the system was successful in the detection and classification of strokes. FastDTW and CNN have shown the highest accuracy for each class and overall average accuracy. Since our system is time-sensitive to provide real-time feedback we depended on FastDTW. This algorithm has ideal or near-optimal synchronization, with $\mathrm{O}(\mathrm{N})$ time and memory complexity. It is an estimated time and frequency algorithm. However, the dependence of CNNs on the initial parameter tuning to avoid local optimal, this mostly computationally expensive because it has to take a large dataset for training. We also conducted a confusion matrix on our chosen algorithm FastDTW ro clarify more our results as shown in table 2 . Which was built on the same data used in table 1 . Moreover, the player's mistakes decreased during the usage of our system, and this is due to feedback in real-time the system provides for the player of the mistakes made in each stroke and overall performance of the session.

\section{Future work and conclusion}

In brief, during this study, we have proposed a framework to train table tennis players and increase their performance. The 
Table 2. Confusion matrix on FastDTW algorithm

\begin{tabular}{|c|c|c|c|c|c|c|c|}
\hline & CFD & CBD & CFTS & WFD & WBD & WFTS & PRECISON \\
\hline CFD & 47 & 0 & 0 & 4 & 0 & 0 & $92.157 \%$ \\
\hline CBD & 0 & 49 & 0 & 0 & 2 & 0 & $96.078 \%$ \\
\hline CFTS & 0 & 0 & 47 & 0 & 0 & 3 & $94 \%$ \\
\hline WFD & 3 & 0 & 0 & 45 & 0 & 0 & $93.75 \%$ \\
\hline WBD & 0 & 1 & 0 & 1 & 48 & 1 & $94.11 \%$ \\
\hline WFTS & 0 & 0 & 3 & 0 & 0 & 46 & $93.878 \%$ \\
\hline Recall & $94 \%$ & $98 \%$ & $94 \%$ & $90 \%$ & $96 \%$ & $92 \%$ & \\
\hline
\end{tabular}

system was built on the basics of strokes in table tennis.

Moreover, by the utilization of an IR depth camera, the system acknowledges the mistakes made by the player in his/her playing technique. In addition, the usage of AR was very useful and was not either distributive or uncomfortable. Based on the experiments tested using different classification algorithms and sensors on various players, we have concluded the system was able to increase the player's performance by $66 \%$. Our future work targets adding extra advances to the framework informational index, normalize, and get with an advanced level in utilizing the AR.

\section{References}

[1] C. Andrews, "Sports tech: Table tennis technology," May 2017. [On-line]. Available: https://eandt.theiet.org/content/articles/2017/05/sportstech-table-tennis-technology/

[2] S. Lock, "Table tennis: number of participants U.S. 2017," Feb. $2017 . \quad$ [On-line]. Available: https://www.statista.com/statistics/191959/participants-intable-tennis-in-the-us-since-2006/

[3] Ben Larcombe. The four basic table tennis strokes, Sep 2018.

[4] Killertips Network, L. Sharon, and W. Suwito. Table Tennis Killer Tips: National Team Edition. Killer tips. Killer tips Network, 2019.

[5] H. Hegazy, M. Abdelsalam, M. Hussien, S. Elmosalamy, Y. M. Hassan, A. M. Nabil and A. Atia, "Online detection and classification of in-corrected played strokes in table tennis using IR depth camera," Procedia Computer Science, vol. 170, pp. 555 - 562, 2020. https://doi.org/10.1016/j.procs.2020.03.125

[6] Habiba Hegazy, Mohamed Abdelsalam, Moustafa Hussien, Seif Elmosalamy, Yomna M.I. Hassan, Ayman M. Nabil, Ayman Atia. "IPingPong: A Real-time Performance Analyzer System for Table Tennis Stroke's Movements," Procedia Computer Science, vol. 175, pp. 80 - 87, 2020. https://doi.org/10.1016/j.procs.2020.07.014

[7] E. Boyer, F. Bevilacqua, F. Phal, and S. Hanneton, "Low-cost motion sensing of table tennis players for real time feedback," Int. J. Table Tennis Sci., vol. 8, 012013.

[8] P. Blank, J. Hossbach, D. Schuldhaus, and B. Eskofier, "Sensor-based stroke detection and stroke type classification in table tennis," 09 2015, pp. 93-100.

https://doi.org/10.1145/2802083.2802087

[9] M. Kos, J. `Zenko, D. Vlaj, and I. Kramberger, "Tennis stroke detection and classification using miniature wearable IMU device," $05 \quad 2016$. https://doi.org/10.1109/IWSSIP.2016.7502764

[10] P. Blank, B. H. Groh, and B. M. Eskofier, "Ball speed and spin estimation in table tennis using a racket-mounted inertial sensor," in Proceedings of the 2017 ACM International Symposium on Wearable Computers. New York, NY, USA: Association for Computing Machinery, 2017, p. 2-9. [Online]. Available: https://doi.org/10.1145/3123021.3123040

[11] J. Tebbe, L. Klamt, Y. Gao, and A. Zell, "Spin detection in robotic table tennis," 2019. https://doi.org/10.1109/ICRA40945.2020.9196536

[12] M. Popa, "Hand gesture recognition based on accelerometer sensors,"01 2011.

[13] R. Liu, Z. Wang, X. Shi, H.-Y. Zhao, S. Qiu, J. Li, and N. Yang, Table Tennis Stroke Recognition Based on Body Sensor Network, 11 2019, pp. 1-10. https://doi.org/10. $\underline{\text { 1007/978-3-030-34914-1_1 }}$

[14] A. Atia and N. Shorim, "Hand gestures classification with multi-core dtw," pp. 91-96, 082019.

[15] Y. Chen, B. Luo, Y.-L. Chen, G. Liang, and X. Wu, “A real-time dynamic hand gesture recognition system using Kinect sensor," 2015 IEEE International Conference on Robotics and Biomimetics (ROBIO), pp. 2026-2030, 2015. https://doi.org/10.1109/ROBIO.2015.7419071

[16] H.-S. Yeo, H. Koike, and A. Quigley, "Augmented learning for sports using wearable head-worn and wristworn devices," 03 2019, pp. 1578-1580. https://doi.org/10.1109/VR.2019.8798054

[17] E. Protopapadakis, A. Grammatikopoulou, A. Doulamis, and G. Nikos, "Folk dance pattern recognition over depth images acquired via Kinect sensor," ISPRS - International Archives of the Photogrammetry, Remote Sensing and Spatial Information Sciences, vol. XLII-2/W3, pp. 587593, 02 2017. https://doi.org/10.5194/isprs-archives-XLII2-W3-587-2017 
[18] T. Le, M.-Q. Nguyen, and T.-M. Nguyen, "Human posture recognition using human skeleton provided by Kinect," 01 2013. https://doi.org/10.1109/ComManTel.2013.6482417

[19] C. Sielu zycki, P. Kaczmarczyk, J. Sobecki, K. Witkowski, J. Maslinski, and W. Cieslinski, "Microsoft Kinect as a tool to support training in professional sports: Augmented reality application to tachi-waza techniques in judo," 09 2016, pp. 153-158. https://doi.org/10.1109/ENIC.2016.030

[20] S. Triamlumlerd, M. Pracha, P. Kongsuwan, and P. Angsuchotmetee, "A table tennis performance analyzer via a single-view low-quality camera," 03 2017, pp. 1-4. https://doi.org/10.1109/IEECON.2017.8075888

[21] A. Chatterjee and V. Govindu, "Noise in structured-light stereo depth cameras: Modeling and its applications," 05 2015.

[22] G. Li, H. Tang, Y. Sun, J. Kong, G. Jiang, D. Jiang, B. Tao, S. Xu, and H. Liu, "Hand gesture recognition based on convolution neural network," Cluster Computing, 12 2017. https://doi.org/10.1007/s10586-017-1435-x

[23] D.-Y. Huang, W.-C. Hu, and S.-H. Chang, "Gabor filterbased hand-pose angle estimation for hand gesture recognition under varying illumination," Expert Syst. Appl., vol. 38, pp. 6031-6042, 052011. https://doi.org/10.1016/j.eswa.2010.11.016

[24] N. Nuttaitanakul and T. Leauhatong, "A novel algorithm for detection human falling from accelerometer signal using wavelet transform and neural network," 10 2015, pp. 215-220. https://doi.org/10.1109/ICITEED.2015.7408944

[25] P. Das, K. Chakravarty, A. Chowdhury, D. Chatterjee, A. Sinha, and A. Pal, "Improving joint position estimation of Kinect using anthropometric constraint based adaptive Kalman filter for rehabilitation," Biomedical Physics and Engineering Express, vol. 4, 12 2017. https://doi.org/10.1088/2057-1976/aaa371

[26] C. Kownacki, "Optimization approach to adapt Kalman filters for the real-time application of accelerometer and gyroscope signal' filtering," Digital Signal Processing, vol. 21, pp. 131-140, 012011. https://doi.org/10.1016/j.dsp.2010.09.001

[27] W. Viyanon, V. Kosasaeng, S. Chatchawal, and A. Komonpetch, "Swingpong: analysis and suggestion based on motion data from mobile sensors for table tennis strokes using decision tree," 12 2016, pp. 1-6. https://doi.org/10.1145/3028842.3028860

[28] C. Xu, P. H. Pathak, and P. Mohapatra, "Finger-writing with smartwatch: A case for finger and hand gesture recognition using smartwatch," in HotMobile '15, 2015. https://doi.org/10.1145/2699343.2699350

[29] S. Canavan, W. Keyes, R. Mccormick, J. Kunnumpurath, T. Hoelzel,and L. Yin, "Hand gesture recognition using a skeleton-based feature representation with a random regression forest," 09 2017, pp. 2364-2368. https://doi.org/10.1109/ICIP.2017.8296705

[30] E. Wu and H. Koike, "Futurepong: Real-time table tennis trajectory forecasting using pose prediction network," in Extended Abstracts of the 2020 CHI Conference on Human Factors in Computing Systems, ser. CHI EA '20. New York, NY, USA: Association for
Computinghttps://doi.org/10.1145/3334480.3382853

Machinery, 2020, p. 1-8. [Online]. Available: https://doi.org/10.1145/3334480.3382853

[31] P. Molchanov, X. Yang, S. Gupta, K. Kim, S. Tyree, and J. Kautz, "Online detection and classification of dynamic hand gestures with recurrent $3 \mathrm{~d}$ convolutional neural networks," 06 2016, pp. 4207-4215. https://doi.org/10.1109/CVPR.2016.456

[32] S. Sempena, N. Maulidevi, and P. Aryan, "Human action recognition using dynamic time warping," 07 2011, pp. 15. https://doi.org/10.1109/ICEEI.2011.6021605

[33] I. Pernek, K. A. Hummel, and P. Kokol, "Exercise repetition detection for resistance training based on smartphones," Personal and Ubiquitous Computing, vol. 17, pp. 771-782, 2012. https://doi.org/10.1007/s00779$\underline{012-0626-y}$

[34] F. Normanton, I. Ardiyanto, and S. Wibirama, "Light sport exercise detection based on smartwatch and smartphone using k-nearest neighbour and dynamic time warping algorithm," 10 2016, pp. 1-5. https://doi.org/10.1109/ICITEED.2016.7863299

[35] A. Switonski, H. Josinski, and K. Wojciechowski, "Dynamic time warping in classification and selection of motion capture data," Multidimensional Systems and Signal Processing, vol. 30, no. 3, pp. 1437-1468, Jul 2019. https://doi.org/10.1007/s11045-018-0611-3

[36] J. Raheja and A. Chaudhary, "Robust gesture recognition using Kinect: A comparison between dtw and hmm," Optik - International Journal for Light and Electron Optics, 01 2015. https://doi.org/10.1016/j.ijleo.2015.02.043

[37] S. Salvador and P. Chan, "Toward accurate dynamic time warping in linear time and space," intel. Data Anal., vol. 11, pp. 561-580, 10 2007. https://doi.org/10.3233/IDA2007-11508

[38] A. Yasser, D. Tariq, R. Samy, M. Allah, and A. Atia, "Smart coaching: Enhancing weightlifting and preventing injuries," International Journal of Advanced Computer Science and Applications, vol. 10, 012019. https://doi.org/10.14569/IJACSA.2019.0100789

[39] S. Zollmann, T. Langlotz, M. Loos, W. H. Lo, and L. Baker, "Arspectator: Exploring augmented reality for sport events," in SIGGRAPH Asia 2019 Technical Briefs, ser. SA '19. New York, NY, USA: Association for Computing Machinery, 2019, p. 75-78. https://doi.org/10.1145/3355088.3365162

[40] X. Gao, J. Tian, X. Liang, and G. Wang, “Arpp: An augmented reality3d ping-pong game system on android mobile platform," 05 2014, pp.1-6. https://doi.org/10.1109/WOCC.2014.6839917

[41] K. Adams, A. Kiefer, D. Panchuk, A. Hunter, R. MacPherson, and W. Spratford, "From the field of play to the laboratory: Recreating the demands of competition with augmented reality simulated sport," Journal of Sports $\begin{array}{lllll}\text { Sciences, } & \text { vol. } & 38, & 12 & 2019 .\end{array}$ https://doi.org/10.1080/02640414.2019.1706872

[42] D. O’Neill, “Enhancing winter sport activities: Improving the visual perception and spatial awareness of downhill 
winter athletes with augmented reality headset displays," 2019.

[43] N. Romli, A. Razali, N. H. Ghazali, N. Hanin, and S. Ibrahim, "Mobile augmented reality (AR) marker-based for indoor library navigation," IOPConference Series: Materials Science and Engineering, vol. 767, p.012062, 03 2020. https://doi.org/10.1088/1757-899X/767/1/012062

[44] S. Khalid, T. Khalil, and S. Nasreen, "A survey of feature selection and feature extraction techniques in machine learning," Proceedings of2014 Science and Information Conference, SAI 2014, pp. 372-378, 102014. https://doi.org/10.1109/SAI.2014.6918213

[45] Devineau, Guillaume \& Xi, W \& Moutarde, Fabien \& Yang, J. (2019). Convolutional Neural Networks for Multivariate Time Series Classification using both Interand Intra- Channel Parallel Convolutions. 